\title{
A new coronavirus associated with human respiratory disease in China
}

https://doi.org/10.1038/s41586-020-2008-3

Received: 7 January 2020

Accepted: 28 January 2020

Published online: 3 February 2020

\section{Open access}

Check for updates

\author{
Fan $\mathrm{Wu}^{1,7}$, Su Zhao ${ }^{2,7}$, Bin $\mathrm{Yu}^{3,7}$, Yan-Mei Chen ${ }^{1,7}$, Wen Wang ${ }^{4,7}$, Zhi-Gang Song ${ }^{1,7}, \mathrm{Yi} \mathrm{Hu}^{2,7}$, \\ Zhao-Wu Tao' ${ }^{2}$, Jun-Hua Tian ${ }^{3}$, Yuan-Yuan Pei', Ming-Li Yuan' ${ }^{2}$, Yu-Ling Zhang', Fa-Hui Dai', \\ Yi Liu', Qi-Min Wang', Jiao-Jiao Zheng ', Lin Xu' , Edward C. Holmes ${ }^{1,5}$ \& Yong-Zhen Zhang ${ }^{1,4,6 凶}$
}

Emerging infectious diseases, such as severe acute respiratory syndrome (SARS) and Zika virus disease, present a major threat to public health ${ }^{1-3}$. Despite intense research efforts, how, when and where new diseases appear are still a source of considerable uncertainty. A severe respiratory disease was recently reported in Wuhan, Hubei province, China. As of 25January 2020, at least 1,975 cases had been reported since the first patient was hospitalized on 12 December 2019. Epidemiological investigations have suggested that the outbreak was associated with a seafood market in Wuhan. Here we study a single patient who was a worker at the market and who was admitted to the Central Hospital of Wuhan on 26 December 2019 while experiencing a severe respiratory syndrome that included fever, dizziness and a cough. Metagenomic RNA sequencing ${ }^{4}$ of a sample of bronchoalveolar lavage fluid from the patient identified a new RNA virus strain from the family Coronaviridae, which is designated here 'WH-Human 1' coronavirus (and has also been referred to as '2019-nCoV'). Phylogenetic analysis of the complete viral genome (29,903 nucleotides) revealed that the virus was most closely related (89.1\% nucleotide similarity) to a group of SARS-like coronaviruses (genus Betacoronavirus, subgenus Sarbecovirus) that had previously been found in bats in $\mathrm{China}^{5}$. This outbreak highlights the ongoing ability of viral spill-over from animals to cause severe disease in humans.
The patient studied was a 41-year-old man with no history of hepatitis, tuberculosis or diabetes. He was admitted to and hospitalized in the Central Hospital of Wuhan on 26 December 2019, 6 days after the onset of disease. The patient reported fever, chest tightness, unproductive cough, pain and weakness for 1 week on presentation (Table 1). Physical examination of cardiovascular, abdominal and neurological characteristics was that these were normal. Mild lymphopoenia (defined as less than $9 \times 10^{5}$ cells per $\mathrm{ml}$ ) was observed, but white blood cell and blood platelet counts were normal in a complete blood count test. Elevated levels of C-reactive protein $\left(41.4 \mathrm{mg} \mathrm{l}^{-1}\right.$ of blood; reference range, $0-6 \mathrm{mg} \mathrm{l}^{-1}$ ) were observed and the levels of aspartate aminotransferase, lactic dehydrogenase and creatine kinase were slightly elevated in blood chemistry tests. The patient had mild hypoxaemia with oxygen levels of $67 \mathrm{~mm} \mathrm{Hg}$ as determined by an arterial blood gas test. On the first day of admission (day 6 after the onset of disease), chest radiographs were abnormal with air-space shadowing such as ground-glass opacities, focal consolidation and patchy consolidation in both lungs (Extended Data Fig. 1). Computedtomography scans of the chest revealed bilateral focal consolidation, lobar consolidation and patchy consolidation, especially in the lower lung (Extended Data Fig. 1a-d). A chest radiograph revealed a bilateral diffuse patchy and fuzzy shadow on day 5 after admission (day 11 after the onset of disease) (Extended Data Fig. 1e). Preliminary aetiological investigations excluded the presence of influenza virus, Chlamydia pneumoniae and Mycoplasma pneumoniae using commercial pathogen antigen-detection kits, and this was confirmed by PCR. Other common respiratory pathogens, including human adenoviruses, also tested negative by quantitative PCR (qPCR) (Extended Data Fig. 2). Although a combination of antibiotic, antiviral and glucocorticoid therapy was administered, the patient exhibited respiratory failure and was given high-flow non-invasive ventilation. The condition of the patient did not improve after 3 days of treatment and he was admitted to the intensive care unit. The patient was transferred to another hospital in Wuhan for further treatment 6 days after admission.

Epidemiological investigations by the Wuhan Center for Disease Control and Prevention revealed that the patient worked at a local indoor seafood market. Notably, in addition to fish and shellfish, a variety of live wild animals-including hedgehogs, badgers, snakes and birds (turtledoves)-were available for sale in the market before the outbreak began, as well as animal carcasses and animal meat. No bats were available for sale. While the patient might have had contact with wild animals at the market, he recalled no exposure to live poultry.

To investigate the possible aetiological agents associated with this disease, we collected bronchoalveolar lavage fluid (BALF) and

${ }^{1}$ Shanghai Public Health Clinical Center, Fudan University, Shanghai, China. ${ }^{2}$ Department of Pulmonary and Critical Care Medicine, The Central Hospital of Wuhan, Tongji Medical College, Huazhong University of Science and Technology, Wuhan, China. ${ }^{3}$ Wuhan Center for Disease Control and Prevention, Wuhan, China. ${ }^{4}$ Department of Zoonosis, National Institute for

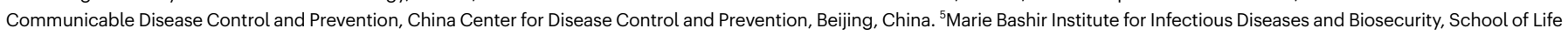
and Environmental Sciences and School of Medical Sciences, The University of Sydney, Sydney, New South Wales, Australia. ${ }^{6}$ School of Public Health, Fudan University, Shanghai, China.

${ }^{7}$ These authors contributed equally: Fan Wu, Su Zhao, Bin Yu, Yan-Mei Chen, Wen Wang, Zhi-Gang Song, Yi Hu. ${ }^{\circledR e}$-mail: zhangyongzhen@shphc.org.cn 


\section{Table 1 | Clinical symptoms and patient data}

\begin{tabular}{ll}
\hline Characteristic & Patient \\
\hline Age (years) & 41 \\
\hline Sex & Male \\
\hline Date of illness onset & 20 December 2019 \\
\hline Date of admission & 26 December 2019 \\
\hline Signs and symptoms & Yes \\
\hline Fever & 38.4 \\
\hline Body temperature $\left({ }^{\circ} \mathrm{C}\right)$ & Yes \\
\hline Cough & Yes \\
\hline Sputum production & Yes \\
\hline Dizzy & Yes \\
\hline Weakness & Yes \\
\hline Chest tightness & Yes \\
\hline Dyspnoea & Negative \\
\hline Bacterial culture & Yes \\
\hline Glucocorticoid therapy & Cefoselis \\
\hline Antibiotic therapy & Oseltamivir \\
\hline Antiviral therapy & Mechanical ventilation \\
\hline Oxygen therapy & \\
\hline
\end{tabular}

performed deep meta-transcriptomic sequencing. The clinical specimen was handled in a biosafety level 3 laboratory at Shanghai Public Health Clinical Center. Total RNA was extracted from $200 \mu \mathrm{l}$ of BALF and a meta-transcriptomic library was constructed for pairend (150-bp reads) sequencing using an Illumina MiniSeq as previously described ${ }^{4,6-8}$. In total, we generated $56,565,928$ sequence reads that were de novo-assembled and screened for potential aetiological agents. Of the 384,096 contigs assembled by Megahit ${ }^{9}$, the longest $(30,474$ nucleotides $(\mathrm{nt}))$ had a high abundance and was closely related to a bat SARS-like coronavirus (CoV) isolate-bat SL-CoVZC45 (GenBank accession number MG772933)-that had previously been sampled in China, with a nucleotide identity of $89.1 \%$ (Supplementary Tables 1, 2). The genome sequence of this virus, as well as its termini, were determined and confirmed by reverse-transcription PCR (RT-PCR $)^{10}$ and $5^{\prime} / 3^{\prime}$ rapid amplification of cDNA ends (RACE), respectively. This virus strain was designated as WH-Human 1 coronavirus (WHCV) (and has also been referred to as ' $2019-\mathrm{nCoV}^{\prime}$ ') and its whole genome sequence $(29,903 \mathrm{nt})$ has been assigned GenBank accession number MN908947. Remapping the RNA-sequencing data to the complete genome of WHCV resulted in an assembly of 123,613 reads, providing $99.99 \%$ genome coverage at a mean depth of $6.04 \times($ range, 0.01-78.84 $\times$ ) (Extended Data Fig. 3). The viral load in the BALF sample was estimated by qPCR to be $3.95 \times 10^{8}$ copies per $\mathrm{ml}$ (Extended Data Fig. 4 ).

The viral genome organization of WHCV was determined by sequence alignment to two representative members of the genus Betacoronavirus: a coronavirus associated with humans (SARS-CoV Tor2, GenBank accession number AY274119) and a coronavirus associated with bats (bat SL-CoVZC45, GenBank accession number MG772933). The untranslational regions and open-reading frame (ORF) of WHCV were mapped on the basis of this sequence alignment and ORF prediction. The WHCV viral genome was similar to these two coronaviruses (Fig. 1 and Supplementary Table 3). The order of genes ( $5^{\prime}$ to $\left.3^{\prime}\right)$ was as follows: replicase $O R F 1 a b$, spike $(S)$, envelope $(E)$, membrane $(M)$ and nucle$\operatorname{ocapsid}(N)$. WHCV has $5^{\prime}$ and $3^{\prime}$ terminal sequences that are typical of betacoronaviruses, with $265 \mathrm{nt}$ at the $5^{\prime}$ terminal end and $229 \mathrm{nt}$ at the $3^{\prime}$ terminal end. The predicted replicase ORF1ab gene of WHCV is $21,291 \mathrm{nt}$ in length and contained 16 predicted non-structural proteins (Supplementary Table 4), followed by (at least) 13 downstream ORFs. Additionally, WHCV shares a highly conserved domain (LLRKNGNKG: amino acids 122-130) in $n s p 1$ with SARS-CoV. The predicted $S, O R F 3 a$, $E, M$ and $N$ genes of WHCV are 3,822, 828, 228, 669 and 1,260 nt in length, respectively. In addition to these ORF regions, which are shared by all members of the subgenus Sarbecovirus, WHCV is similar to SARS-CoV in that it carries a predicted $O R F 8$ gene (with a length of $366 \mathrm{nt}$ ) that is located between the $M$ and $N O R F$ genes. The functions of WHCV ORFs were predicted on the basis of those of known coronaviruses and are described in Supplementary Table 5. In a manner similar to SARS-CoV Tor2, a leader transcription regulatory sequence (TRS) and nine putative body TRSs could be readily identified upstream of the $5^{\prime}$ end of the ORF in WHCV, and the putative conserved TRS core sequence appeared in two forms-ACGAAC or CUAAAC (Supplementary Table 6).

To determine the evolutionary relationships between WHCV and previously identified coronaviruses, we estimated phylogenetic trees on the basis of the nucleotide sequences of the whole-genome sequence, the non-structural protein genes ORF1 $a$ and $O R F 1 b$, and the main structural proteins encoded by the $S, E, M$ and $N$ genes (Fig. 2 and Extended Data Fig. 5). In all phylogenies, WHCV clustered with members of the subgenus Sarbecovirus, including the SARS-CoV that was responsible for the global SARS pandemic ${ }^{1,2}$ of 2002-2003, as well as a number

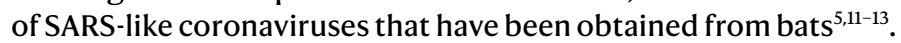
However, WHCV changed topological position within the subgenus Sarbecovirus depending on which gene was used, which suggests that recombination has occurred in this group of viruses in the past (Fig. 2 and Extended Data Fig. 5). Specifically, in the $S$ gene tree (Extended Data Fig. 5), WHCV was most closely related to the bat coronavirus SL-CoVZC45 with $82.3 \%$ amino acid identity (and around $77.2 \%$ amino acid identity to SARS-CoV; Supplementary Table 3) whereas in the
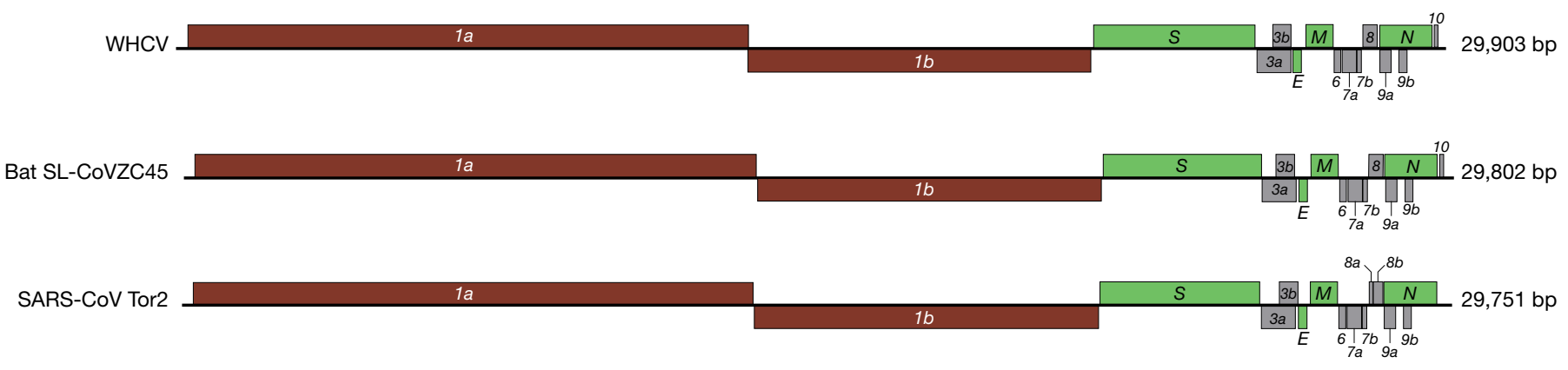

\begin{tabular}{|c|c|c|c|c|c|c|c|c|c|c|c|c|c|}
\hline 1 & 1 & 1 & 1 & 1 & 1 & 1 & 1 & 1 & 1 & & 1 & 1 & \\
\hline 2,000 & 4,000 & 6,000 & 8,000 & 10,000 & 12,000 & 14,000 & 16,000 & 18,000 & 20,000 & 22,000 & 24,000 & 26,000 & 28,000 \\
\hline
\end{tabular}

Fig. 1 Genome organization of SARS and SARS-like CoVs. The organization of genes for WHCV, bat SL-CoVZC45 and SARS-CoV Tor2. 

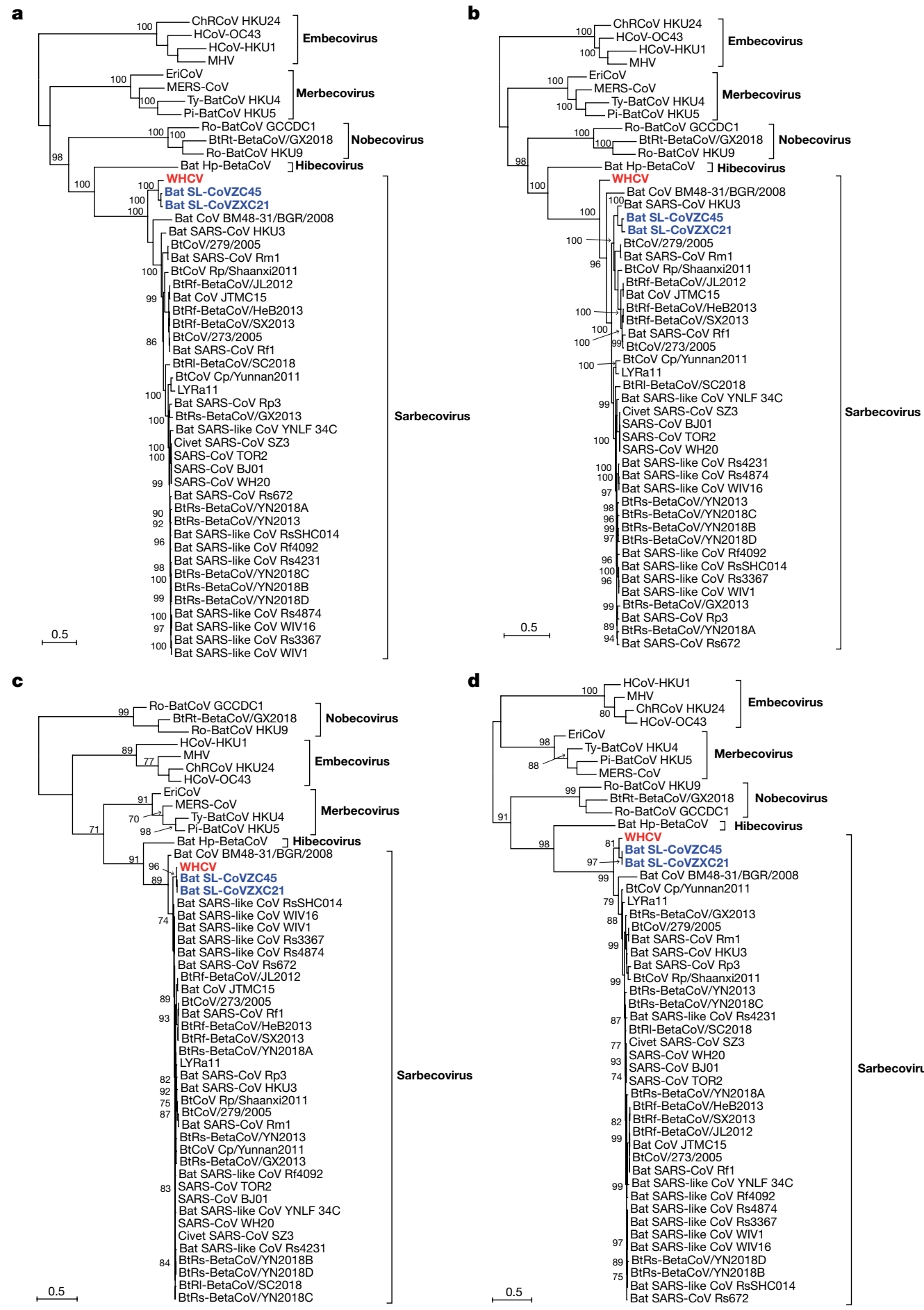

Fig. 2 | Maximum likelihood phylogenetic trees of nucleotide sequences of the ORF1a, ORF1b, E and $M$ genes of WHCV and related coronaviruses.

a, Phylogenetic trees of ORF1a. b, Phylogenetic trees of ORF1b.c, Phylogenetic trees of $E$.d, Phylogenetic trees of $M$. EriCoV, Erinaceus coronavirus. Numbers

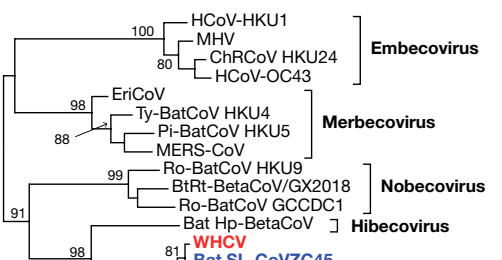

81 [ Bat SL-CovZC45

99 Bat CoV BM48-31/BGR/2008

BtCoV Cp/Yunnan2011

79 LYRa11

$88[$ BtRs-BetaCoV/GX

99 | Bat SARS-CoV Rm1

Bat SARS-CoV Rp3

99 [ $\mathrm{BtCoV}$ Rp/Shaanxi2011

BtRs-BetaCoV/YN2013
BtRs-BetaCoV/YN2018C

87 Bat SARS-like CoV Rs423

BtRI-BetaCoV/SC2018

SARS-CoV WH20

SARS-CoV BJ01

SARS-CoV TOR2

BtRs-BetaCoV/YN2018A

BtRf-BetaCoV/HeB2013

BtRf-BetaCoV/SX2013
BtRf-BetaCoV/JL2012

Bat CoV JTMC15

BtCoV/273/2005

Bat SARS-CoV Rf1

99 Bat SARS-like CoV YNLF $34 \mathrm{C}$

Bat SARS-like CoV Rf4092

Bat SARS-like CoV Rs4874

Bat SARS-like CoV WIV1

Bat SARS-like CoV WIV16

89 BtRs-BetaCoV/YN2018D

75 BtRs-BetaCoV/YN2018B

0.5

Bat SARS-like CoV RsS
Bat SARS-CoV Rs672

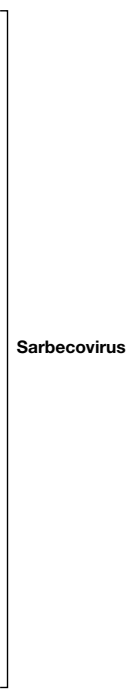

(>70) above or below the branches indicate percentage bootstrap values for the associated nodes. The trees were mid-point rooted for clarity only. The scale bar represents the number of substitutions per site.

ORF1b phylogeny, WHCV fell in a basal position within the subgenus Sarbecovirus (Fig. 2). This topological division, which probably reflects recombination among the bat sarbecoviruses, was also observed in the phylogenetic trees estimated for conserved domains in the replicase polyprotein pplab (Extended Data Fig. 6).

To better understand the potential of WHCV to infect humans, the receptor-binding domain (RBD) of its spike protein was compared

with those of SARS-CoVs and bat SARS-like CoVs. The RBD sequences of WHCV were more closely related to those of SARS-CoVs $(73.8-74.9 \%$ amino acid identity) and SARS-like CoVs, including strains Rs4874, Rs7327 and Rs4231 (75.9-76.9\% amino acid identity), that are able to use the human ACE2 receptor for cell entry ${ }^{11}$ (Supplementary Table 7). In addition, the RBD of the spike protein from WHCV was only one amino acid longer than the RBD of the spike protein from SARS-CoV (Extended 


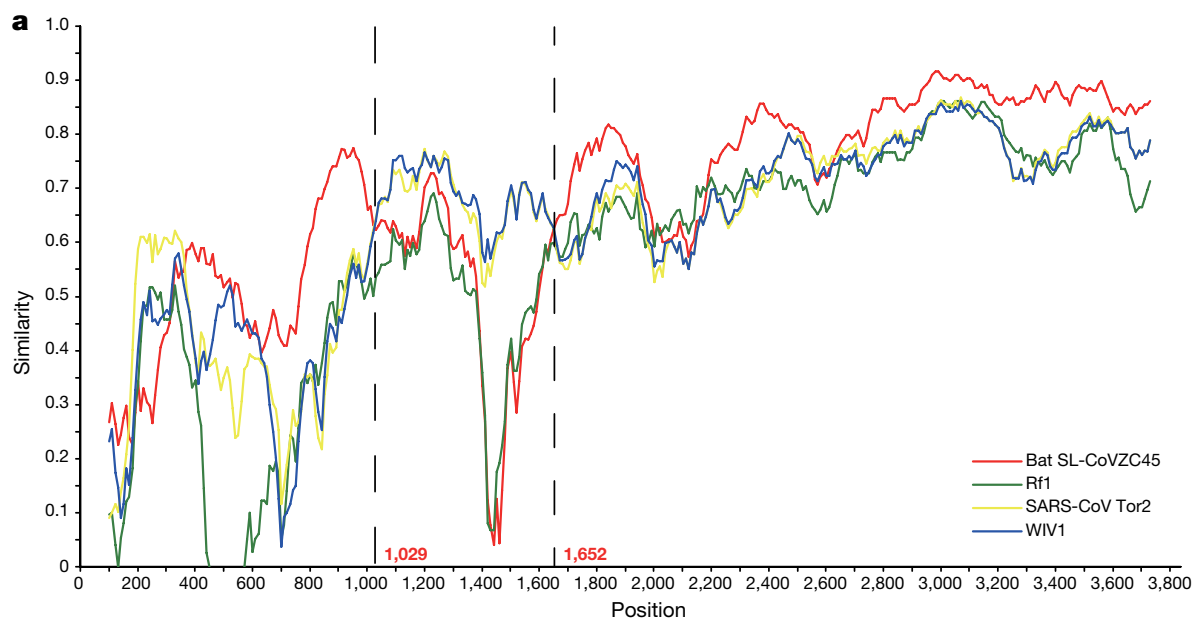

b
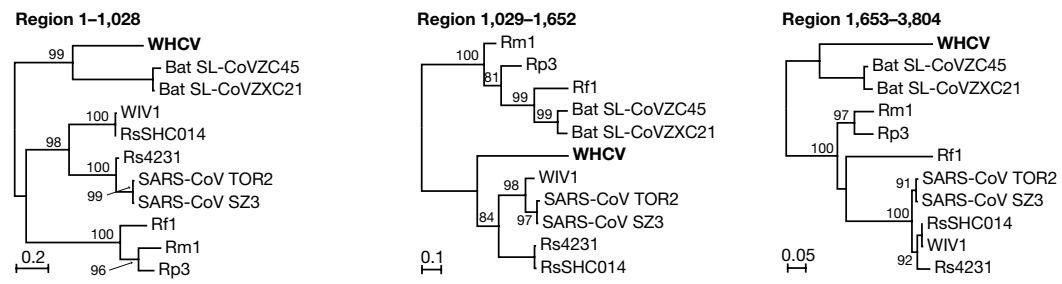

Fig. $3 \mid$ Possible recombination events in the $S$ gene of sarbecoviruses. a, The sequence similarity plot reveals two putative recombination breakpoints (black dashed lines), with their locations indicated at the bottom. The plot shows similarity comparisons of the $S$ gene of WHCV (query) compared with the sequences of SARS-CoV Tor 2 and bat SARS-like CoVs WIV1, Rf 1 and
CoVZC45. b, Phylogenies of the major parental region (1-1,028 and 1,653$3,804)$ and minor parental region $(1,029-1,652)$. Phylogenies were estimated using a maximum likelihood method and were mid-point rooted for clarity only. Numbers above or below the branches indicate percentage bootstrap values. The scale bar represents the number of substitutions per site.
Data Fig. 7a). By contrast, other bat SARS-like CoVs-including the Rp3 strain that cannot bind to human ACE2 ${ }^{14}$-had amino acid deletions at positions 433-437 and 460-472 compared with the sequence in SARS-CoVs (Extended Data Fig. 7a). The previously determined ${ }^{15}$ crystal structure of the RBD of the spike protein of SARS-CoV complexed with human ACE2 (Protein Data Bank (PDB) 2AJF) revealed that regions 433-437 and 460-472 directly interact with human ACE2 and hence may be important in determining species specificity (Extended Data Fig. 7b). We predicted the three-dimensional protein structures of the RBD domains of the spike protein of WHCV, Rs4874 and Rp3 by protein homology modelling using the SWISS-MODEL server and compared them to the crystal structure of RBD domain of the spike protein of SARS-CoV (PDB 2GHV) (Extended Data Fig. 7c-f). In accordance with the sequence alignment, the predicted protein structures of the RBD domains of WHCV and Rs4874 were closely related to that of SARS-CoV and different from the predicted structure of the RBD domain from Rp3. In addition, the N terminus of the spike protein of WHCV is more similar to that of SARS-CoV than other human coronaviruses (HKU1 and OC43) (Extended Data Fig. 8) that can bind to sialic acid ${ }^{16}$. In summary, the high similarities of the amino acid sequences and predicted protein structures of the RBD domains of WHCV and SARS-CoV suggest that WHCV may efficiently use human ACE2 as a receptor for cellular entry, which could potentially facilitate human-to-human transmis$\operatorname{sion}^{11,17,18}$.

To further characterize the putative recombination events in the evolutionary history of the sarbecoviruses, the whole-genome sequence of WHCV and four representative coronaviruses-bat SARS-like CoV Rp3, CoVZC45, CoVZXC21 and SARS-CoV Tor2-were analysed using the Recombination Detection Program v.4 (RDP4) ${ }^{19}$. Although the similarity plots suggested that possible recombination events had occurred between WHCV and SARS-CoVs or SARS-like CoVs (Extended Data Fig. 9), there was no significant evidence for recombination across the genome as a whole. However, some evidence for past recombination was detected in the $S$ gene of WHCV, SARS-CoV and bat SARS-like CoVs (WIV1 and RsSHC014) $\left(P<3.147 \times 10^{-3}\right.$ to $\left.P<9.198 \times 10^{-9}\right)$, for which the similarity plots suggested the presence of recombination breakpoints at nucleotides 1,029 and 1,652, which separate the $S$ gene of WHCV into three regions (Fig. 3). In phylogenies of the nucleotide fragments from 1 to 1,029 and from 1,652 to the end of the sequence, WHCV was most closely related to bat SL-CoVZC45 and bat SL-CoVZXC21, whereas in the region of nucleotides 1,030 to 1,651 (the RBD region) WHCV grouped with SARS-CoV and bat SARS-like CoVs (WIV1 and RsSHC014) that are capable of direct human transmission ${ }^{17,20}$. Despite these recombination events, which seem relatively common among sarbecoviruses, there is no evidence that recombination has facilitated the emergence of WHCV.

Coronaviruses are associated with a number of infectious disease outbreaks in humans, including SARS in 2002-2003 and Middle East respiratory syndrome (MERS) in $2012^{1,21}$. Four other coronaviruseshuman coronaviruses HKU1, OC43, NL63 and 229E-are also associated with respiratory disease ${ }^{22-25}$. Although SARS-like coronaviruses have been widely identified in mammals including bats since 2005 in China $^{10,26-28}$, the exact origin of human-infected coronaviruses remains unclear. Here we describe a new coronavirus-WHCV-in the BALF from a patient who experienced severe respiratory disease in Wuhan, China. Phylogenetic analysis suggests that WHCV is a member of the genus Betacoronavirus (subgenus Sarbecovirus) that has some genomic and phylogenetic similarities to SARS-CoV ${ }^{1}$, particularly in the RBD of the spike protein. These genomic and clinical similarities to SARS, as well as its high abundance in clinical samples, provides evidence for an association between WHCV and the ongoing outbreak of respiratory disease in Wuhan and across the world. Although the isolation of the virus from only a single patient is not sufficient to conclude that it caused these respiratory symptoms, our findings have been independently corroborated in further patients in a separate study ${ }^{29}$. 
The identification of multiple SARS-like CoVs in bats have led to the idea that these animals act as hosts of a natural reservoir of these viruse $^{22,23}$. Although SARS-like viruses have been identified widely in bats in China, viruses identical to SARS-CoV have not yet been documented. Notably, WHCV is most closely related to bat coronaviruses, and shows $100 \%$ amino acid similarity to bat SL-CoVZC45 in the nsp7 and E proteins (Supplementary Table 3). Thus, these data suggest that bats are a possible host for the viral reservoir of WHCV. However, as a variety of animal species were for sale in the market when the disease was first reported, further studies are needed to determine the natural reservoir and any intermediate hosts of WHCV.

Note added in proof: Since this paper was accepted, the ICTV has designated the virus as SARS-CoV-2 ${ }^{30}$; in addition, the WHO has released the official name of the disease caused by this virus, which is COVID-19 ${ }^{31}$.

\section{Online content}

Any methods, additional references, Nature Research reporting summaries, source data, extended data, supplementary information, acknowledgements, peer review information; details of author contributions and competing interests; and statements of data and code availability are available at https://doi.org/10.1038/s41586-020-2008-3.

1. Drosten, C. et al. Identification of a novel coronavirus in patients with severe acute respiratory syndrome. N. Engl. J. Med. 348, 1967-1976 (2003).

2. Wolfe, N. D., Dunavan, C. P. \& Diamond, J. Origins of major human infectious diseases. Nature 447, 279-283 (2007).

3. Ventura, C. V., Maia, M., Bravo-Filho, V., Góis, A. L. \& Belfort, R. Jr. Zika virus in Brazil and macular atrophy in a child with microcephaly. Lancet 387, 228 (2016).

4. Shi, M. et al. Redefining the invertebrate RNA virosphere. Nature 540, 539-543 (2016).

5. Hu, D. et al. Genomic characterization and infectivity of a novel SARS-like coronavirus in Chinese bat. Emerg. Microbes Infect. 7, 1-10 (2018).

6. Shi, M. et al. The evolutionary history of vertebrate RNA viruses. Nature 556, 197-202 (2018).

7. Yadav, P. D. et al. Nipah virus sequences from humans and bats during Nipah outbreak, Kerala, India, 2018. Emerg. Infect. Dis. 25, 1003-1006 (2019).

8. McMullan, L. K. et al. Characterisation of infectious Ebola virus from the ongoing outbreak to guide response activities in the Democratic Republic of the Congo: a phylogenetic and in vitro analysis. Lancet Infect. Dis. 19, 1023-1032 (2019).

9. Li, D., Liu, C. M., Luo, R., Sadakane, K. \& Lam, T. W. MEGAHIT: an ultra-fast single-node solution for large and complex metagenomics assembly via succinct de Bruijn graph. Bioinformatics 31, 1674-1676 (2015).

10. Wang, W. et al. Discovery, diversity and evolution of novel coronaviruses sampled from rodents in China. Virology 474, 19-27 (2015).

11. Hu, B. et al. Discovery of a rich gene pool of bat SARS-related coronaviruses provides new insights into the origin of SARS coronavirus. PLoS Pathog. 13, e1006698 (2017).

12. Lin, X.-D. et al. Extensive diversity of coronaviruses in bats from China. Virology 507, 1-10 (2017).

13. $\mathrm{Xu}$, L. et al. Detection and characterization of diverse alpha- and betacoronaviruses from bats in China. Virol. Sin. 31, 69-77 (2016).
14. Ren, W. et al. Difference in receptor usage between severe acute respiratory syndrome (SARS) coronavirus and SARS-like coronavirus of bat origin. J. Virol. 82, 1899-1907 (2008).

15. Li, F., Li, W., Farzan, M. \& Harrison, S. C. Structure of SARS coronavirus spike receptorbinding domain complexed with receptor. Science 309, 1864-1868 (2005).

16. Hulswit, R. J. G. et al. Human coronaviruses OC43 and HKU1 bind to 9-O-acetylated sialic acids via a conserved receptor-binding site in spike protein domain A. Proc. Natl Acad. Sci. USA 116, 2681-2690 (2019).

17. Ge, X. Y. et al. Isolation and characterization of a bat SARS-like coronavirus that uses the ACE2 receptor. Nature 503, 535-538 (2013).

18. Yang, X. L. et al. Isolation and characterization of a novel bat coronavirus closely related to the direct progenitor of severe acute respiratory syndrome coronavirus. J. Virol. 90, 3253-3256 (2016).

19. Martin, D. P. et al. RDP3: a flexible and fast computer program for analyzing recombination. Bioinformatics 26, 2462-2463 (2010).

20. Menachery, V. D. et al. A SARS-like cluster of circulating bat coronaviruses shows potential for human emergence. Nat. Med. 21, 1508-1513 (2015).

21. Bermingham, A. et al. Severe respiratory illness caused by a novel coronavirus, in a patient transferred to the United Kingdom from the Middle East, September 2012. Euro Surveill. 17, 20290 (2012).

22. Hamre, D. \& Procknow, J. J. A new virus isolated from the human respiratory tract. Proc. Soc. Exp. Biol. Med. 121, 190-193 (1966).

23. McIntosh, K., Becker, W. B. \& Chanock, R. M. Growth in suckling-mouse brain of "IBV-like" viruses from patients with upper respiratory tract disease. Proc. Natl Acad. Sci. USA 58, 2268-2273 (1967).

24. van der Hoek, L. et al. Identification of a new human coronavirus. Nat. Med. 10, 368-373 (2004).

25. Woo, P. C. et al. Characterization and complete genome sequence of a novel coronavirus, coronavirus HKU1, from patients with pneumonia. J. Virol. 79, 884-895 (2005).

26. Li, W. et al. Bats are natural reservoirs of SARS-like coronaviruses. Science 310, 676-679 (2005).

27. Lau, S. K. et al. Severe acute respiratory syndrome coronavirus-like virus in Chinese horseshoe bats. Proc. Natl Acad. Sci. USA 102, 14040-14045 (2005).

28. Wang, W. et al. Discovery of a highly divergent coronavirus in the Asian house shrew from China illuminates the origin of the Alphacoronaviruses. J. Virol. 91, e00764-17 (2017).

29. Zhou, P. et al. A pneumonia outbreak associated with a new coronavirus of probable bat origin. Nature https://doi.org/10.1038/s41586-020-2012-7 (2020).

30. Gorbalenya, A. E. Severe acute respiratory syndrome-related coronavirus - the species and its viruses, a statement of the Coronavirus Study Group. Preprint at bioRxiv https:// doi.org/10.1101/2020.02.07.93786 (2020).

31. WHO. WHO Director-General's remarks at the media briefing on 2019-nCoV on 11 February 2020. https://www.who.int/dg/speeches/detail/who-director-general-s-remarksat-the-media-briefing-on-2019-ncov-on-11-february-2020 (WHO, 11 February 2020).

Publisher's note Springer Nature remains neutral with regard to jurisdictional claims in published maps and institutional affiliations.

Open Access This article is licensed under a Creative Commons Attribution 4.0 International License, which permits use, sharing, adaptation, distribution and reproduction in any medium or format, as long as you give appropriate credit to the original author(s) and the source, provide a link to the Creative Commons licence, and indicate if changes were made. The images or other third party material in this article are included in the article's Creative Commons licence, unless indicated otherwise in a credit line to the material. If material is not included in the article's Creative Commons licence and your intended use is not permitted by statutory regulation or exceeds the permitted use, you will need to obtain permission directly from the copyright holder. To view a copy of this licence, visit http://creativecommons.org/licenses/by/4.0/.

(c) The Author(s) 2020 


\section{Methods}

\section{Data reporting}

No statistical methods were used to predetermine sample size. The experiments were not randomized and the investigators were not blinded to allocation during experiments and outcome assessment.

\section{Patient information and collection of clinical data and sample}

A patient presenting with acute onset of fever (temperature over $37.5^{\circ} \mathrm{C}$ ), cough and chest tightness, who was admitted to the Central Hospital of Wuhan, in Wuhan, China, was considered to be a suspected case. During admission, BALF was collected and stored at $-80^{\circ} \mathrm{C}$ until further processing. Demographic, clinical and laboratory data were retrieved from the clinical records of the patient. The study was reviewed and approved by the ethics committee of the National Institute for Communicable Disease Control and Prevention, Chinese Center for Disease Control and Prevention. Signed written informed consent was obtained from the patient.

\section{RNA library construction and sequencing}

Total RNA was extracted from the BALF sample using the RNeasy Plus Universal Mini kit (Qiagen) following the manufacturer's instructions. The quantity and quality of the RNA solution was assessed using a Qbit machine and an Agilent 2100 Bioanalyzer (Agilent Technologies) before library construction and sequencing. An RNA library was then constructed using the SMARTer Stranded Total RNA-Seq kit v.2 (TaKaRa). Ribosomal RNA depletion was performed during library construction following the manufacturer's instructions. Paired-end (150-bp reads) sequencing of the RNA library was performed on the MiniSeq platform (Illumina). Library preparation and sequencing were carried out at the Shanghai Public Health Clinical Center, Fudan University, Shanghai, China.

\section{Data processing and identification of the viral agent}

Sequencing reads were first adaptor and quality trimmed using the Trimmomatic program ${ }^{32}$. The remaining $56,565,928$ reads were assembled de novo using both Megahit (v.1.1.3) ${ }^{9}$ and Trinity (v.2.5.1 ${ }^{33}$ with default parameter settings. Megahit generated a total of 384,096 assembled contigs (size range of 200-30,474 nt), whereas Trinity generated $1,329,960$ contigs with a size range of $201-11,760 \mathrm{nt}$. All of these assembled contigs were compared (using BLASTn and Diamond BLASTx) against the entire non-redundant ( $\mathrm{nr}$ ) nucleotide and protein databases, with $e$ values set to $1 \times 10^{-10}$ and $1 \times 10^{-5}$, respectively. To identify possible aetiological agents present in the sequencing data, the abundance of the assembled contigs was first evaluated as the expected counts using the RSEM program ${ }^{34}$ implemented in Trinity. Non-human reads $(23,712,657$ reads), generated by filtering host reads using the human genome (human release 32, GRCh38.p13, downloaded from Gencode) by Bowtie ${ }^{35}$, were used for the RSEM abundance assessment.

As the longest contigs generated by Megahit $(30,474 \mathrm{nt})$ and Trinity $(11,760 \mathrm{nt})$ both showed high similarity to the bat SARS-like coronavirus isolate bat SL-CoVZC45 and were found at a high abundance (Supplementary Tables 1, 2), the longer sequence (30,474 nt)-which covered almost the whole virus genome-was used for primer design for PCR confirmation and determination of the genome termini. Primers used for PCR, qPCR and RACE experiments are listed in Supplementary Table 8 . The PCR assay was conducted as previously described ${ }^{10}$ and the complete genome termini was determined using the Takara SMARTer RACE 5'/3' kit (TaKaRa) following the manufacturer's instructions. Subsequently, the genome coverage and sequencing depth were determined by remapping all of the adaptor-and quality-trimmed reads to the whole genome of WHCV using Bowtie $2^{35}$ and Samtools ${ }^{36}$.

The viral loads of WHCV in BALF were determined by quantitative real-time RT-PCR using the Takara One Step PrimeScript RT-PCR kit (Takara RR064A) following the manufacturer's instructions. Real-time
RT-PCR was performed using $2.5 \mu$ l RNA with 8 pmol of each primer and 4 pmol probe under the following conditions: reverse transcription at $42^{\circ} \mathrm{C}$ for $10 \mathrm{~min}, 95^{\circ} \mathrm{C}$ for $1 \mathrm{~min}$, followed by 40 cycles of $95^{\circ} \mathrm{C}$ for $15 \mathrm{~s}$ and $60^{\circ} \mathrm{C}$ for $1 \mathrm{~min}$. The reactions were performed and detected by ABI 7500 Real-Time PCR Systems. The PCR product covering the Taqman primers and probe region was cloned into $\mathrm{pLB}$ vector using the Lethal Based Simple Fast Cloning Kit (TianGen) as standards for quantitative viral load test.

\section{Virus genome characterization and phylogenetic analysis}

For the newly identified virus genome, the potential ORFs were predicted and annotated using the conserved signatures of the cleavage sites recognized by coronavirus proteinases, and were processed in the Lasergene software package (v.7.1, DNAstar). The viral genes were aligned using the L-INS-i algorithm implemented in MAFFT (v.7.407) ${ }^{37}$.

Phylogenetic analyses were then performed using the nucleotide sequences of various CoV gene datasets: (1) whole genome, (2) ORF1a, (3) ORF1b, (4) nsp5 (3CLpro), (5) RdRp (nsp12), (6) nsp13 (Hel), (7) nsp14 (ExoN), (8) nsp15 (NendoU), (9) nsp16 (O-MT), (10) spike (S) and (11) nucleocapsid $(\mathrm{N})$. Phylogenetic trees were inferred using the maximum likelihood method implemented in the PhyML program (v.3.0) ${ }^{38}$, using the generalized time reversible substitution model and subtree pruning and regrafting branch swapping. Bootstrap support values were calculated from 1,000 pseudo-replicate trees. The best-fitting model of nucleotide substitution was determined using MEGA $(v .5)^{39}$. Amino acid identities among sequences were calculated using the MegAlign program implemented in the Lasergene software package (v.7.1, DNAstar).

\section{Genome recombination analysis}

Potential recombination events in the history of the sarbecoviruses were assessed using both the RDP $4^{19}$ and Simplot (v.3.5.1) ${ }^{40}$. The RDP4 analysis was conducted based on the complete genome (nucleotide) sequence, using RDP, GENECONV, BootScan, maximum chi square, Chimera, SISCAN and 3SEQ methods. Putative recombination events were identified with a Bonferroni corrected $P$-value cut-off of 0.01 . Similarity plots were inferred using Simplot to further characterize potential recombination events, including the location of possible breakpoints.

\section{Analysis of the RBD domain of the spike protein of WHCV}

An amino acid sequence alignment of RBD sequences from WHCV, SARS-CoVs and bat SARS-like CoVs was performed using MUSCLE ${ }^{41}$. The predicted protein structures of the RBD of the spike protein were estimated based on target-template alignment using ProMod3 on SWISS-MODEL server (https://swissmodel.expasy.org/). The sequences of the RBD domains spike of WHCV, Rs4874 and Rp3 were searched by BLAST against the primary amino acid sequence contained in the SWISS-MODEL template library (last update, 9 January 2020; last included PDB release, 3 January 2020). Models were built based on the target-template alignment using ProMod3. The global and perresidue model quality was assessed using the QMEAN scoring func$\operatorname{tion}^{42}$. The PDB files of the predicted protein structures were displayed and compared with the crystal structures of the spike RBD of SARS-CoV (PDB 2GHV ${ }^{43}$ and the crystal of structure of the spike RBD of SARS-CoV complexed with human ACE2 (PDB 2AJF) ${ }^{15}$.

\section{Reporting summary}

Further information on research design is available in the Nature Research Reporting Summary linked to this paper.

\section{Data availability}

Sequence reads generated in this study are available from the NCBI Sequence Read Archive (SRA) database under BioProject accession 
number PRJNA603194. The complete genome sequence of WHCV has been deposited in GenBank under accession number MN908947.

32. Bolger, A. M., Lohse, M. \& Usadel, B. Trimmomatic: a flexible trimmer for Illumina sequence data. Bioinformatics 30, 2114-2120 (2014).

33. Grabherr, M. G. et al. Full-length transcriptome assembly from RNA-seq data without a reference genome. Nat. Biotechnol. 29, 644-652 (2011).

34. Li, B., Ruotti, V., Stewart, R. M., Thomson, J. A. \& Dewey, C. N. RNA-seq gene expression estimation with read mapping uncertainty. Bioinformatics 26, 493-500 (2010).

35. Langmead, B. \& Salzberg, S. L. Fast gapped-read alignment with Bowtie 2. Nat. Methods 9, 357-359 (2012).

36. Li, H. et al. The Sequence Alignment/Map format and SAMtools. Bioinformatics $\mathbf{2 5}$, 2078-2079 (2009).

37. Katoh, K. \& Standley, D. M. MAFFT multiple sequence alignment software version 7: improvements in performance and usability. Mol. Biol. Evol. 30, 772-780 (2013).

38. Guindon, S. et al. New algorithms and methods to estimate maximum-likelihood phylogenies: assessing the performance of PhyML 3.0. Syst. Biol. 59, 307-321 (2010).

39. Tamura, K. et al. MEGA5: molecular evolutionary genetics analysis using maximum likelihood, evolutionary distance, and maximum parsimony methods. Mol. Biol. Evol. 28, 2731-2739 (2011).

40. Lole, K. S. et al. Full-length human immunodeficiency virus type 1 genomes from subtype C-infected seroconverters in India, with evidence of intersubtype recombination. J. Virol. 73, 152-160 (1999).

41. Edgar, R. C. MUSCLE: multiple sequence alignment with high accuracy and high throughput. Nucleic Acids Res. 32, 1792-1797 (2004).
42. Waterhouse, A. et al. SWISS-MODEL: homology modelling of protein structures and complexes. Nucleic Acids Res. 46, W296-W303 (2018).

43. Hwang, W. C. et al. Structural basis of neutralization by a human anti-severe acute respiratory syndrome spike protein antibody, 80R. J. Biol. Chem. 281, 34610-34616 (2006).

Acknowledgements This study was supported by the Special National Project on investigation of basic resources of China (grant 2019FY101500) and the National Natural Science Foundation of China (grants 81861138003 and 31930001 ). E.C.H. is supported by an ARC Australian Laureate Fellowship (FL170100022).

Author contributions Y.-Z.Z. conceived and designed the study. S.Z., Y.H., Z.-W.T. and M.-L.Y. performed the clinical work and sample collection. B.Y. and J.-H.T. performed the epidemiological investigation and sample collection. F.W., Z.-G.S., L.X., Y.-Y.P., Y.-L.Z., F.-H.D., Y.L., J.-J.Z. and Q.-M.W. performed the experiments. Y.-M.C., W.W., F.W., E.C.H. and Y.-Z.Z. analysed the data. Y.-Z.Z., E.C.H. and F.W. wrote the paper with input from all authors. Y.-Z.Z. led the study.

Competing interests The authors declare no competing interests.

Additional information

Supplementary information is available for this paper at https://doi.org/10.1038/s41586-0202008-3.

Correspondence and requests for materials should be addressed to Y.-Z.Z.

Peer review information Nature thanks Nicholas Loman and the other, anonymous, reviewer(s) for their contribution to the peer review of this work.

Reprints and permissions information is available at http://www.nature.com/reprints. 


\section{Article}
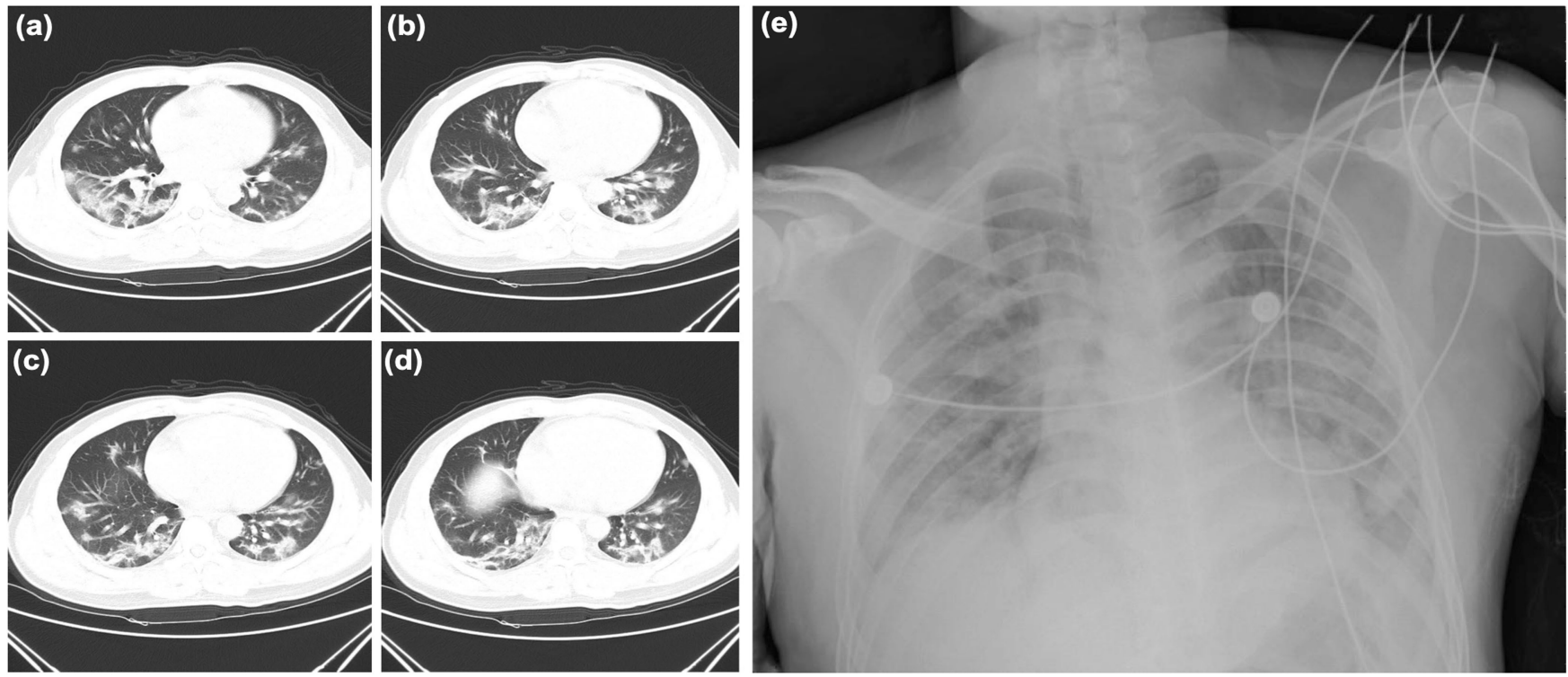

Extended Data Fig. 1 | Chest radiographs of the patient. a-d, Computedtomography scans of the chest were obtained on the day of admission (day 6

and patchy consolidation were clearly observed, especially in the lower lung. e, A chest radiograph was obtained on day 5 after admission (day 11 after the onset of disease). Bilateral diffuse patchy and fuzzy shadows were observed. 
(a)

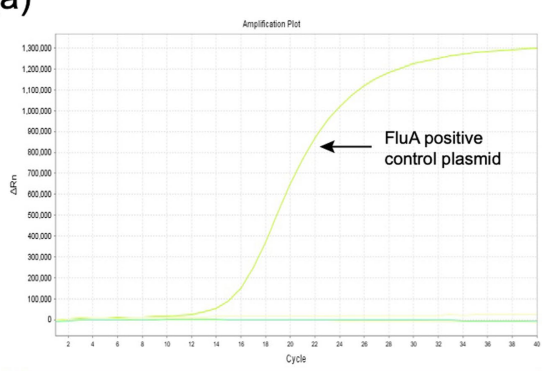

POS III Sample 1 NEG (b)

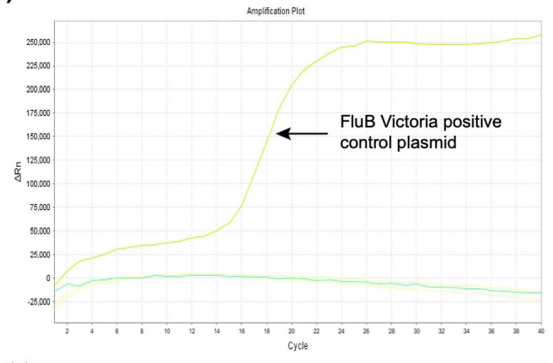

POS II Sample 1 NEG (c)

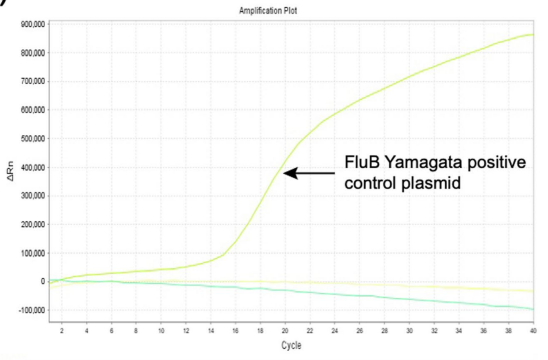

POS II Sample 1 NEG (d)

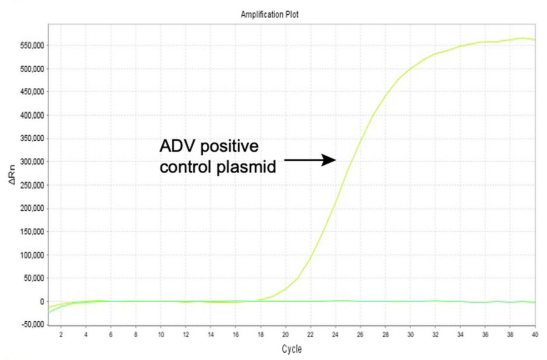

POS III Sample 1 NEG (e)

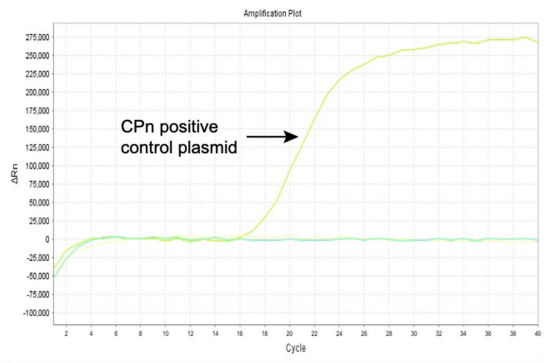

POS III Sample 1 NEG
Extended Data Fig. 2 |Other respiratory pathogens were not detected in the BALF sample by real-time RT-PCR. ate, The BALF sample was tested for the presence of influenza $A$ virus (a), the Victoria lineage of influenza $B$ viruses (b), the Yamagata lineage of influenza B viruses (c), human adenovirus (d) and Chlamydia pneumonia (e). Sample 1 was the BALF sample of the patient, water was used as a negative (NEG) control and positive (POS) control samples included plasmids covering the Taqman primers and probe regions of influenza A, the Victoria and Yamagata lineages of influenza B viruses, human adenovirus and Chlamydia pneumonia. 
Article

Genome coverage: $99.99 \%(29901 \mathrm{nt} / 29903 \mathrm{nt})$ Mean depth: 604.21

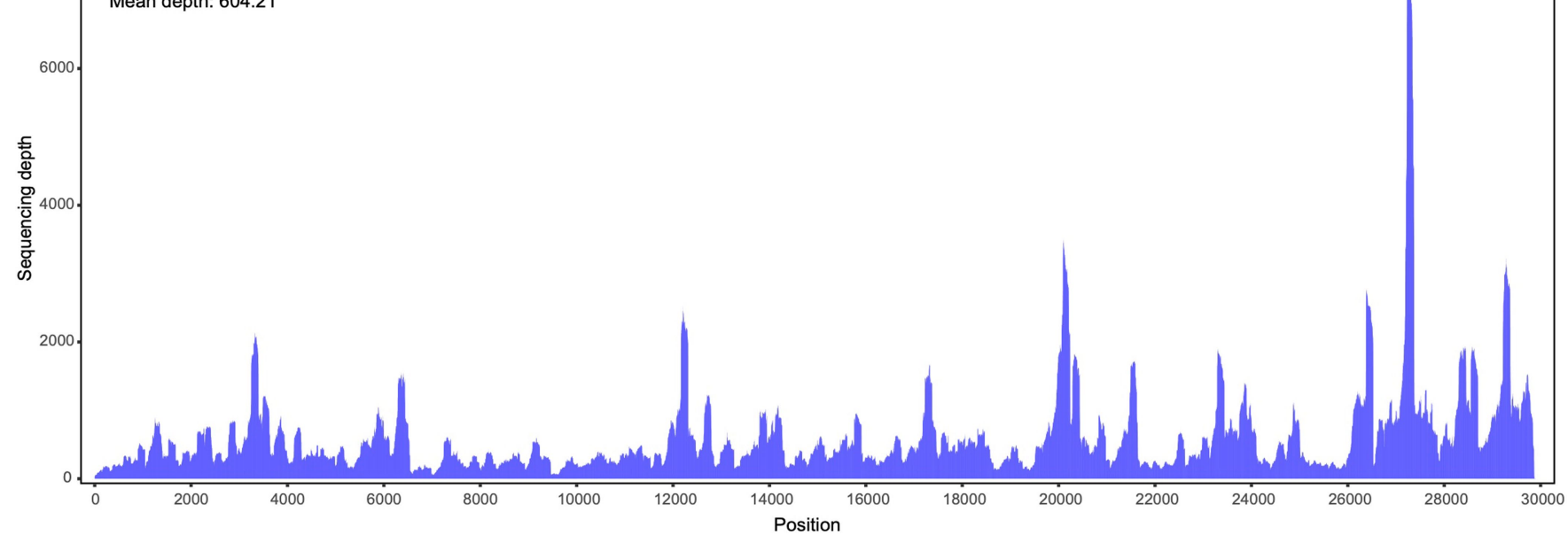

Extended Data Fig. 3 | Mapped read count plot of the WHCV genome. The histograms show the coverage depth per base of the WHCV genome. The mean sequencing depth of the WHCV genome was $604.21 \mathrm{nt}$. 
(a)

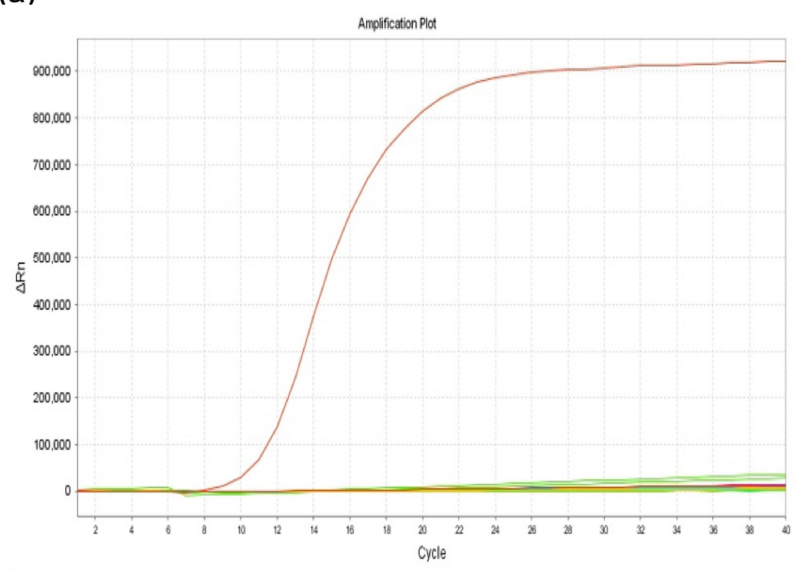

(c)

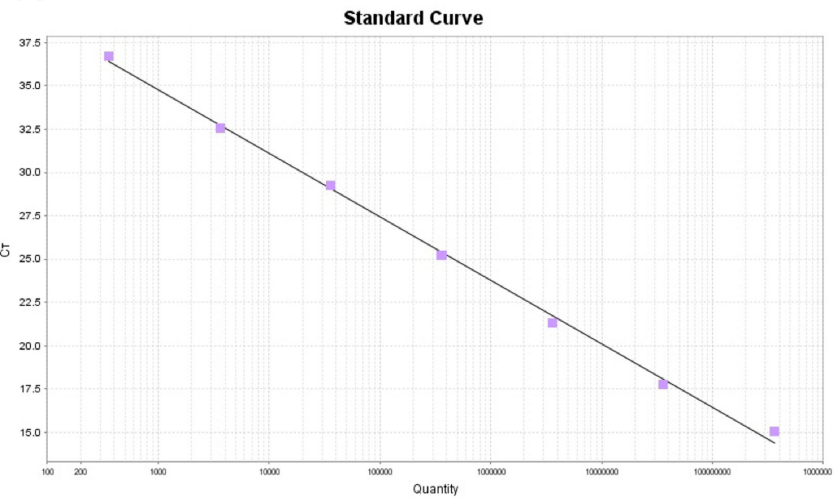

[unt wher

Extended Data Fig. 4 | Quantification of WHCV in clinical samples by realtime RT-PCR. a, Specificity evaluation of the WHCV primers. Test samples comprised clinical samples that were positive for at least one of the following viruses: influenza A virus (09H1N1 and H3N2), influenza B virus, human adenovirus, respiratory syncytial virus, rhinovirus, parainfluenza virus type 1-4, human bocavirus, human metapneumovirus, coronavirus OC43, coronavirus NL63, coronavirus 229E and coronavirus HKU1. Only the standard plasmid of WHCV (WHCV 15,704-16,846 bp in a pLB vector) led to positive (b)

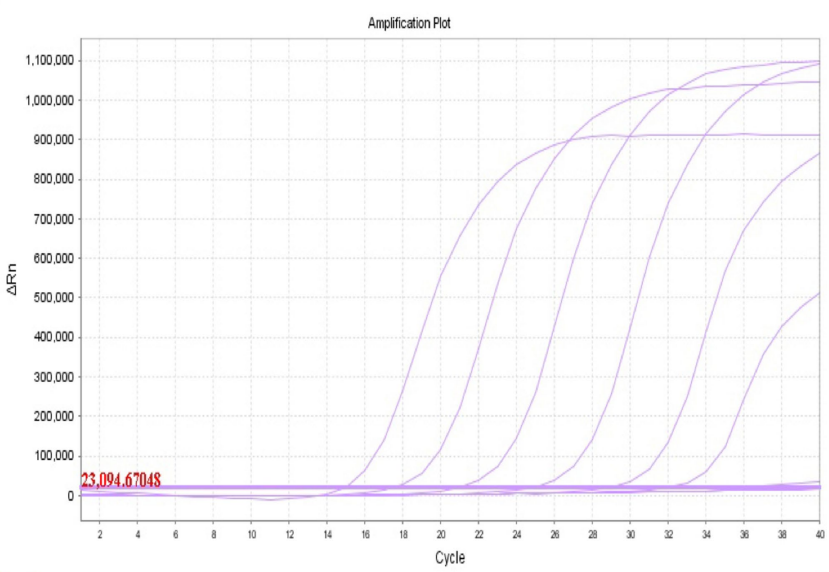

(d)

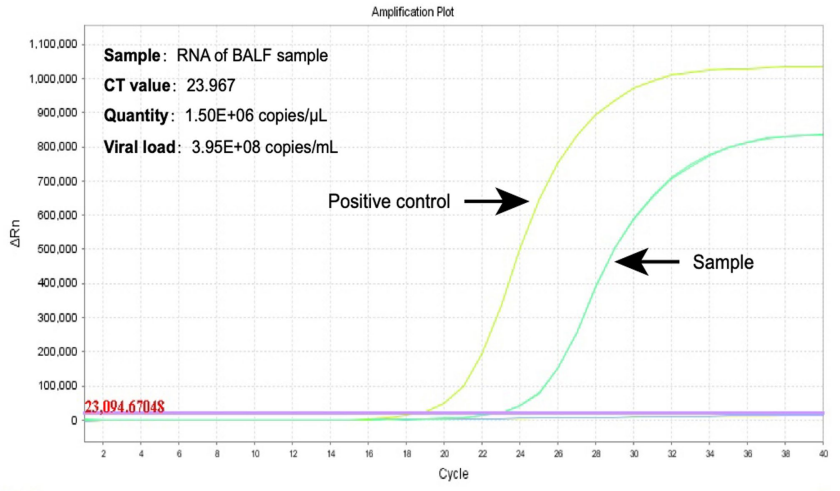

POS NEG $\square$ BLANK $\amalg$ Sample 1

amplification (brown curve). $\mathbf{b}$, Amplification curve of the DNA standard for WHCV. From left to right, the DNA concentrations were $1.8 \times 10^{8}, 1.8 \times 10^{7}$, $1.8 \times 10^{6}, 1.8 \times 10^{5}, 1.8 \times 10^{4}$ and $1.8 \times 10^{3} . \mathrm{c}$, Linear fitted curve of $C_{\mathrm{t}}$ values to concentrations of the WHCV DNA standard. d, Quantification of WHCV in the BALF sample by real-time RT-PCR. The WHCV DNA standard was used as positive control (POS), water (NEG) and blank were used as negative controls. The amplification curve of the BALF sample is shown in green. 
Whole genome

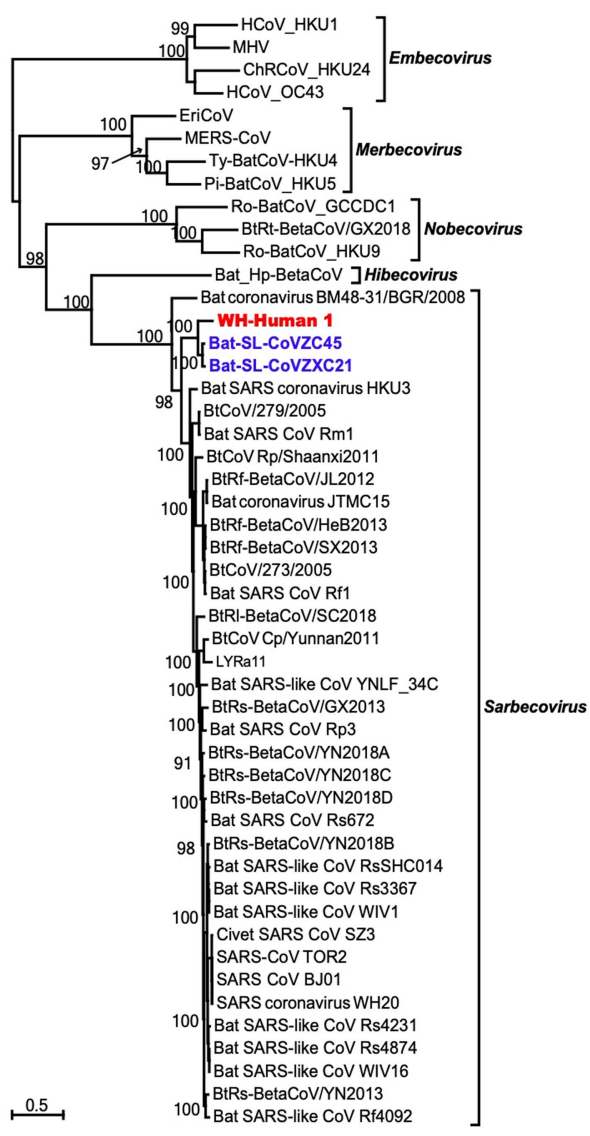

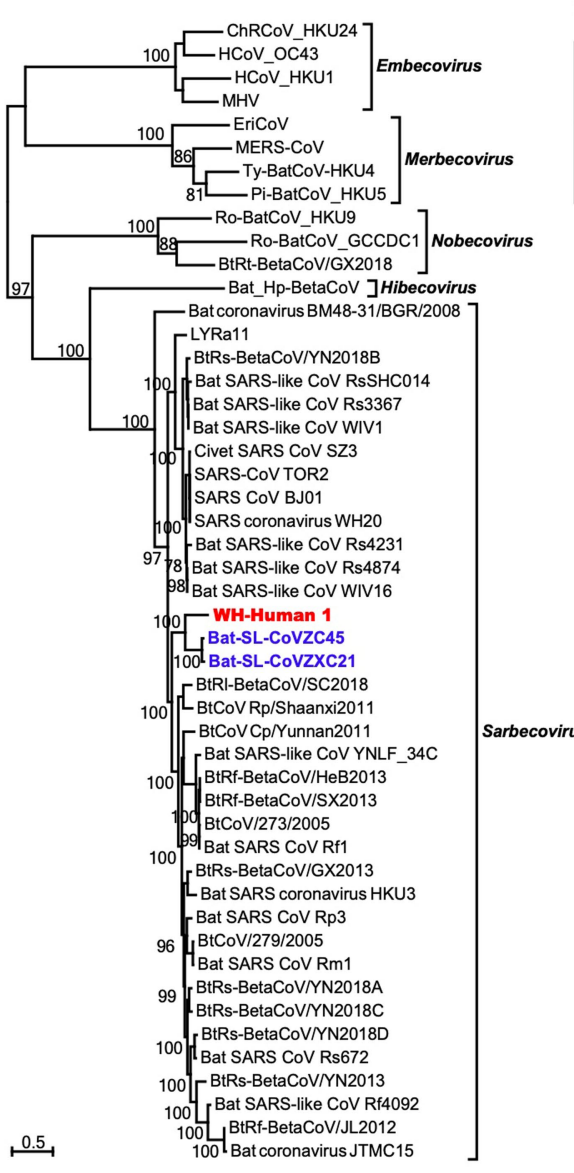

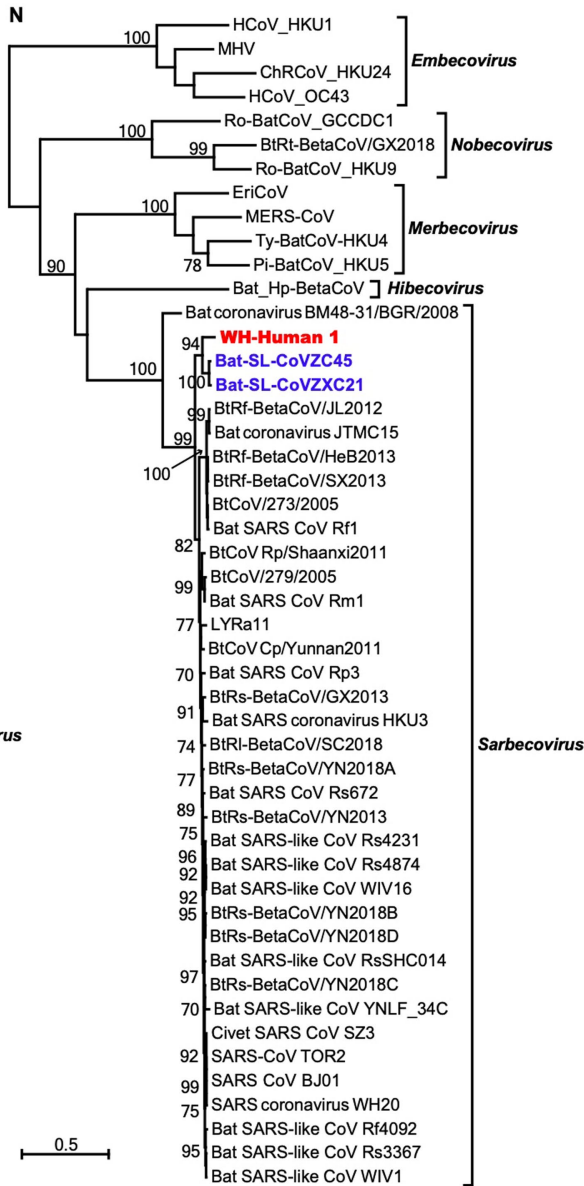

Extended Data Fig. 5 | Maximum likelihood phylogenetic trees of the nucleotide sequences of the whole genome, and $S$ and $N$ genes of WHCV and related coronaviruses. Numbers $(>70)$ above or below the branches indicate percentage bootstrap values. The trees were mid-point rooted for clarity only. The scale bar represents the number of substitutions per site. 


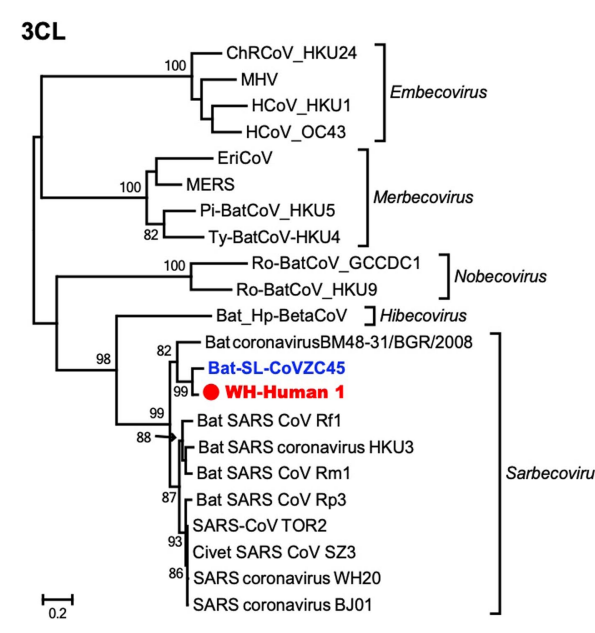

ExoN

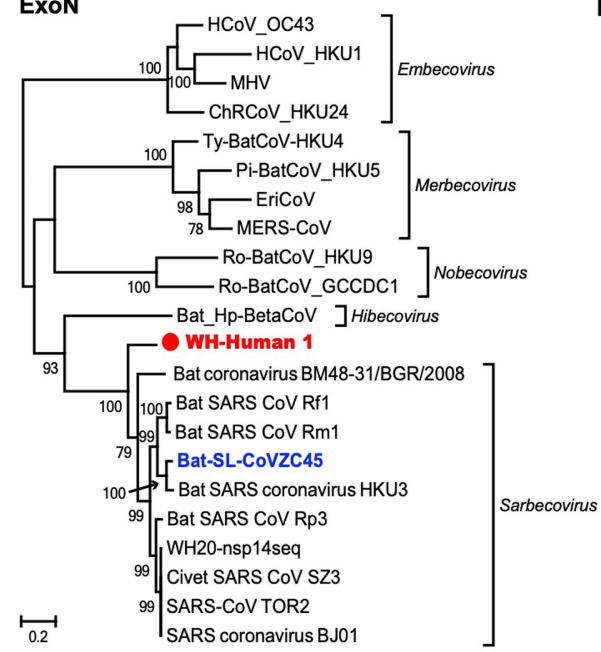

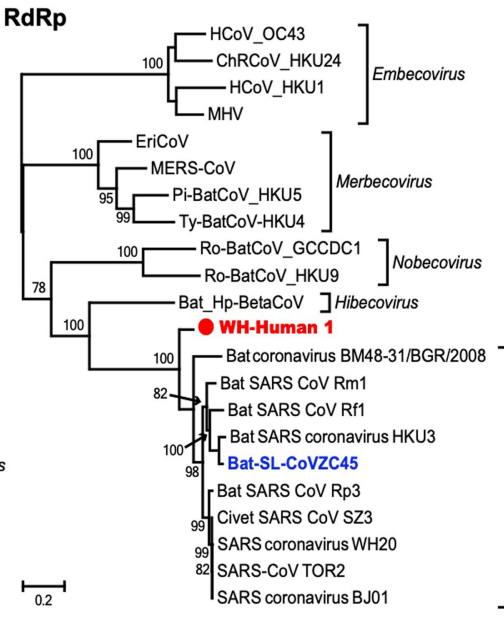

NendoU

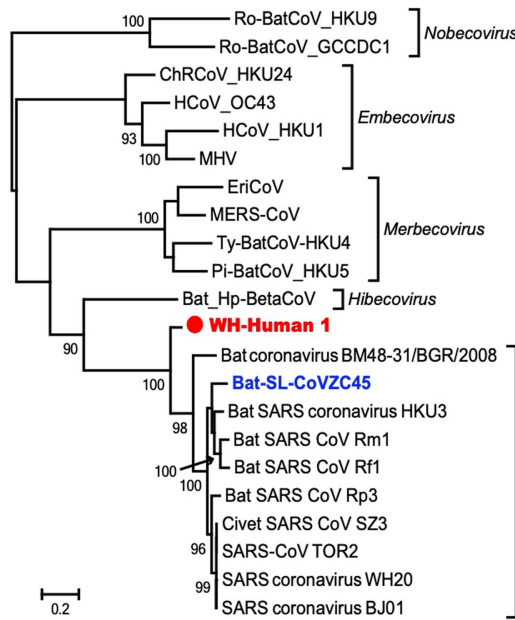

Hel

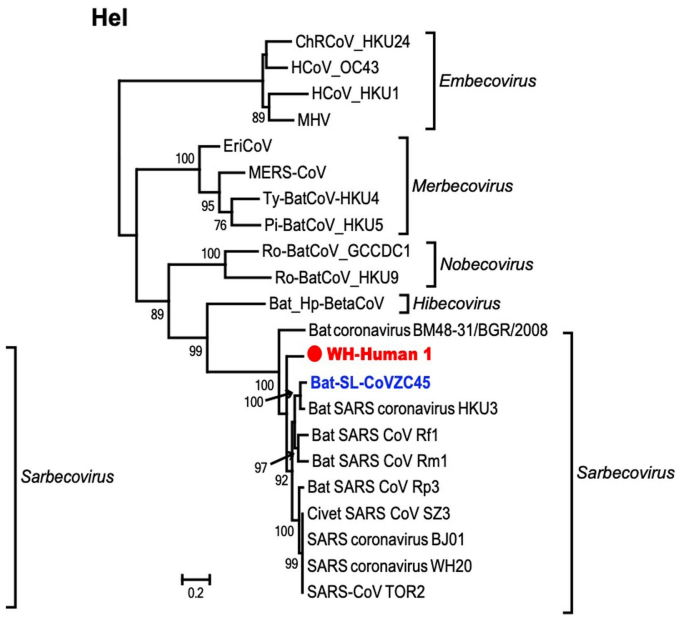

O-MT

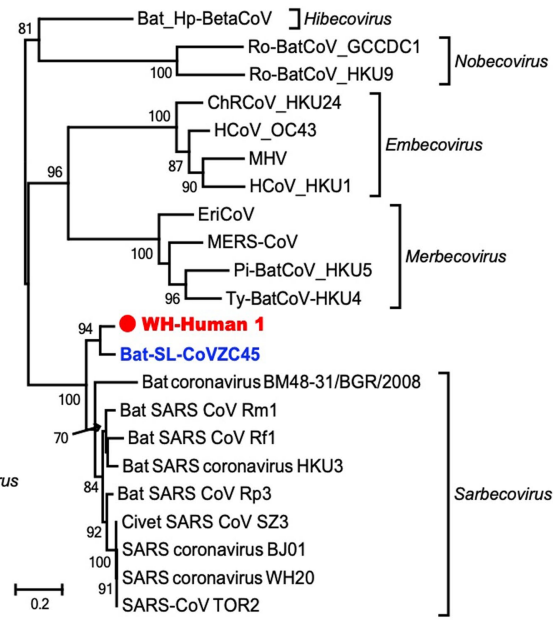

Extended Data Fig. 6 | Maximum likelihood phylogenetic trees of the nucleotide sequences of the $3 C L, R d R p, H e l, E x o N$, NendoU and O-MT genes of WHCV and related coronaviruses. Numbers $(>70)$ above or below the branches indicate percentage bootstrap values. The trees were mid-point rooted for clarity only. The scale bar represents the number of substitutions per site. 
(a)

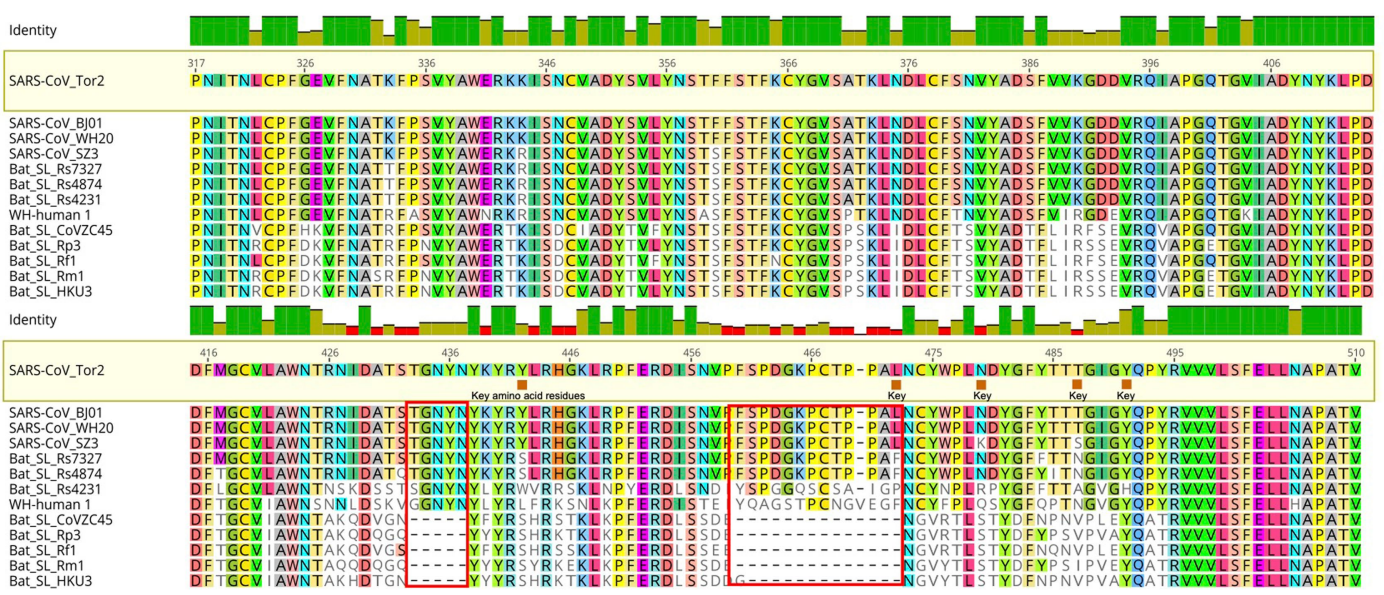

(b)

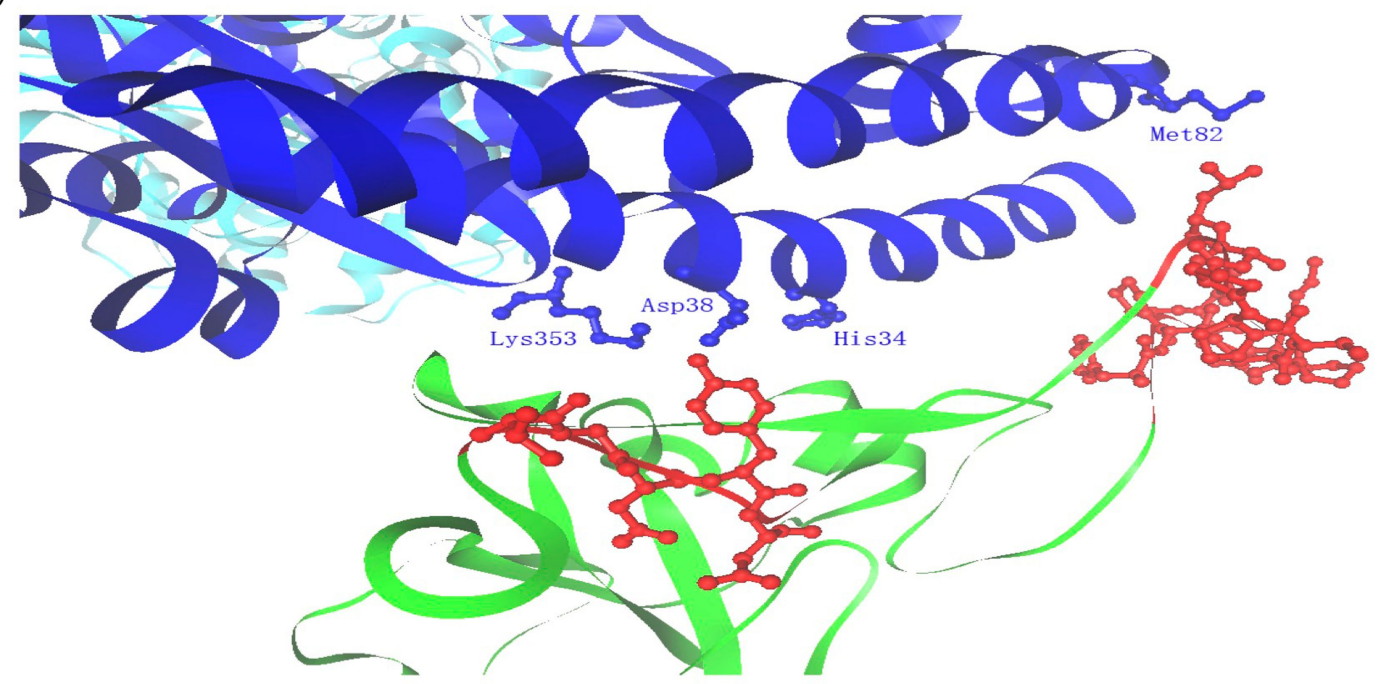

(c)

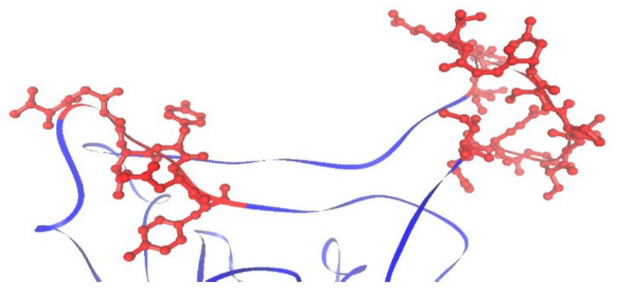

(e)

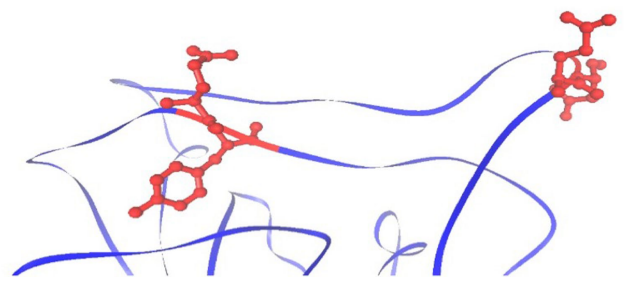

(d)

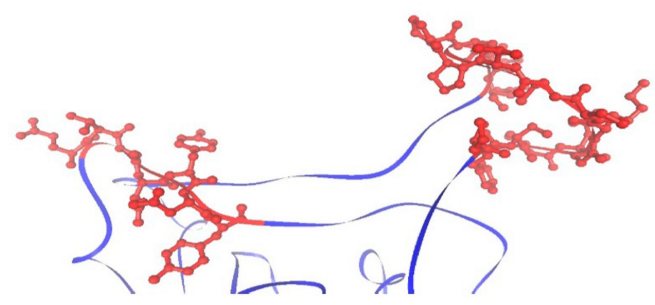

(f)

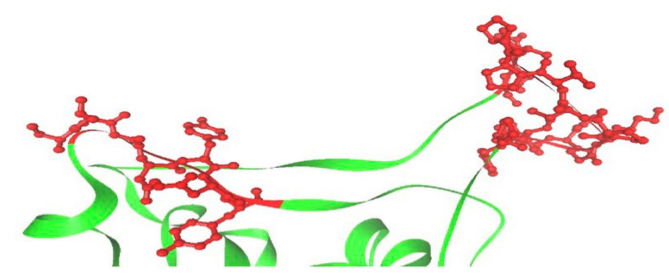

Extended Data Fig. 7 |See next page for caption. 
Extended Data Fig. 7 | Analysis of RBD of the spike protein of WHCV

coronavirus. a, Amino acid sequence alignments of RBD sequences of SARSlike CoVs. Three bat SARS-like CoVs-which could efficiently use the human ACE2 as receptor-had an RBD sequence of similar size to SARS-CoV. WHCV contains a single Val470 insertion. The key amino acid residues involved in the interaction with human ACE2 are marked by orange squares. By contrast, five bat SARS-like CoVs, including Rp3, which has previously been found not to bind to ACE ${ }^{14}$-had amino acid deletions in two motifs (amino acids 433-437 and $460-472$, highlighted by red boxes) compared with those of SARS-CoV.11 b, The two motifs (amino acids 433-437 and 460-472) are shown in red for the crystal structure of the RBD of the spike protein of SARS-CoV in complex with the human ACE2 receptor (PDB 2AJF). Human ACE2 is shown in blue and the RBD of the spike protein of SARS-CoV is shown in green. Important residues in human ACE2 that interact with the RBD of the spike protein of SARS-CoV are marked. c, Predicted protein structure of the RBD of the spike protein of WHCV based on target-template alignment using ProMod3 on the SWISS-MODEL server. d, Predicted structure of the RBD of the spike protein of SARS-like CoV Rs4874. e, Predicted structure of the RBD of the spike protein of SARS-like CoV Rp3. f, Crystal structure of the RBD of the spike protein of SARS-CoV (green) (PDB $2 \mathrm{GHV}$ ). Motifs that resemble amino acids $433-437$ and $460-472$ of the spike protein of SARS-CoV are shown in red. 


\section{Article}

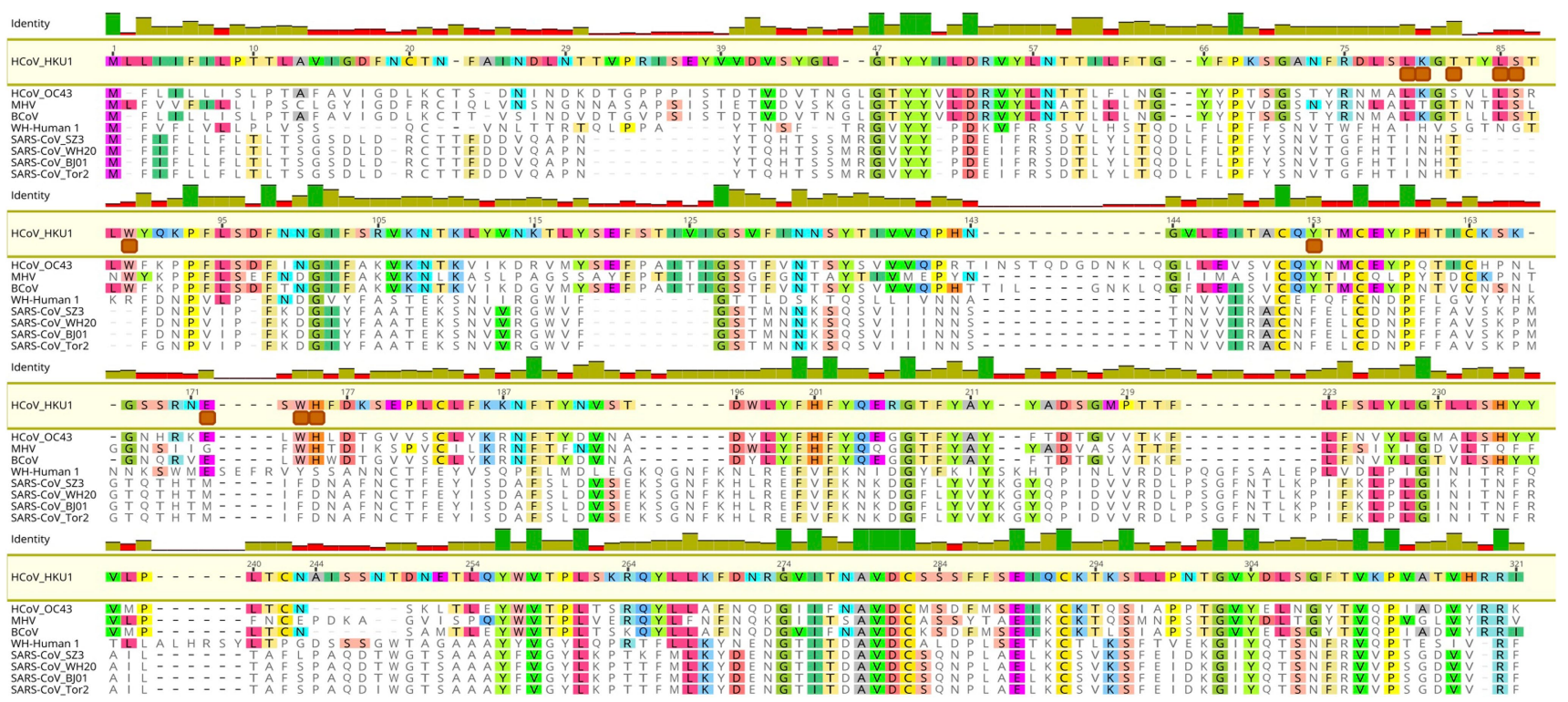

Extended Data Fig. 8 |Amino acid sequence comparison of the $\mathrm{N}$-terminal domain of the spike protein. Amino acid sequence comparison of the $\mathrm{N}$-terminal domain of the spike protein of WHCV, bovine coronavirus (BCoV), mouse hepatitis virus (MHV) and human coronaviruses (HCoV OC43 and HKU1) that can bind to sialic acid and the SARS-CoVs that cannot (SZ3, WH2O, BJO and Tor2). The key residues ${ }^{16}$ for sialic acid binding on $\mathrm{BCoV}$, MHV, and $\mathrm{HCoV} \mathrm{OC} 43$ and HKU1 are highlighted by orange squares. 


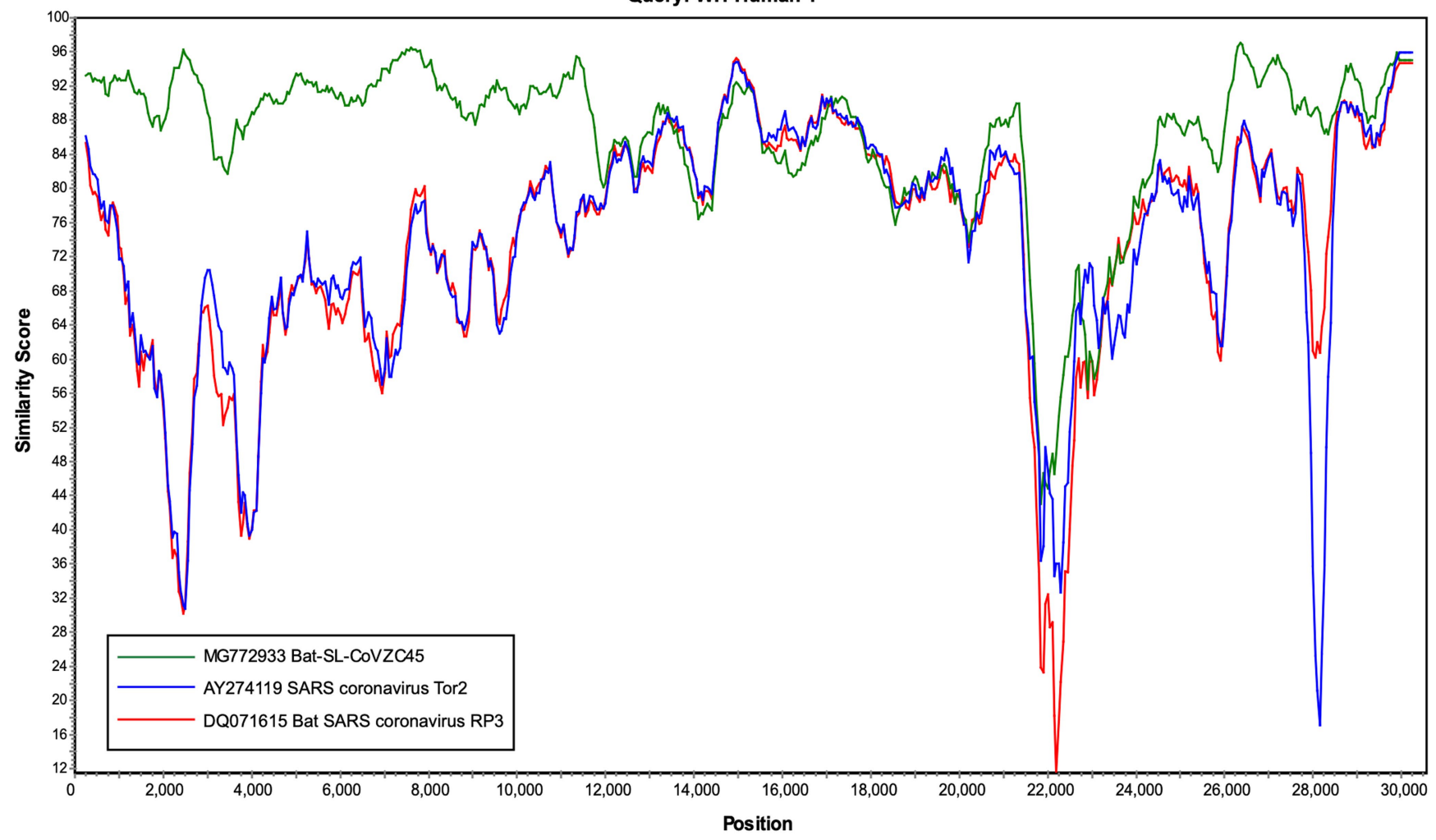

Window : 500 bp, Step: 50 bp, GapStrip: On, Kimura (2-parameter), T/t: 2.0

Extended Data Fig. 9 | Recombination events in WHCV. The sequence similarity plot of WHCV, SARS-like CoVs and bat SARS-like CoVs reveals putative recombination events. 


\section{Reporting Summary}

Nature Research wishes to improve the reproducibility of the work that we publish. This form provides structure for consistency and transparency in reporting. For further information on Nature Research policies, see Authors \& Referees and the Editorial Policy Checklist.

\section{Statistics}

For all statistical analyses, confirm that the following items are present in the figure legend, table legend, main text, or Methods section.

n/a Confirmed

\ $\square$ The exact sample size $(n)$ for each experimental group/condition, given as a discrete number and unit of measurement

Х $\square$ A statement on whether measurements were taken from distinct samples or whether the same sample was measured repeatedly

$\square$ The statistical test(s) used AND whether they are one- or two-sided

$\triangle \square$ Only common tests should be described solely by name; describe more complex techniques in the Methods section.

Х $\square$ A description of all covariates tested

Х $\square$ A description of any assumptions or corrections, such as tests of normality and adjustment for multiple comparisons

$\searrow \square$ A full description of the statistical parameters including central tendency (e.g. means) or other basic estimates (e.g. regression coefficient)

X $\square$ AND variation (e.g. standard deviation) or associated estimates of uncertainty (e.g. confidence intervals)

$\triangle \square$ For null hypothesis testing, the test statistic (e.g. $F, t, r$ ) with confidence intervals, effect sizes, degrees of freedom and $P$ value noted

\ $\square$ Give P values as exact values whenever suitable.

Х $\square$ For Bayesian analysis, information on the choice of priors and Markov chain Monte Carlo settings

Х $\square$ For hierarchical and complex designs, identification of the appropriate level for tests and full reporting of outcomes

Х $\square$ Estimates of effect sizes (e.g. Cohen's $d$, Pearson's $r$ ), indicating how they were calculated

Our web collection on statistics for biologists contains articles on many of the points above.

\section{Software and code}

Policy information about availability of computer code

Data collection

No software was used.

Data analysis

Trimmotic (v0.39): adaptor- and quality-trimming of sequencing reads

Megahit (v1.1.3) and Trinity (v2.5.1): de novo assembly of reads

Blastn (v2.7.1), Diamond blastx (v0.9.21): homology based annotation of sequencing reads and contigs

Bowtie2 (v2.3.4.1) and samtools ( $v 0.1 .19-44428 \mathrm{~cd})$ : read mapping and result analysis

MAFFT (v7.407) and MUSCLE(v3.8.425): sequence alignment

PhyML (v3.0): Phylogenetic tree estimation

MEGA (v5): Best-fit model of nucleotide substitution determination and trees generation

Lasergene software package (v7.1): ORF prediciion and annotation

Geneious prime (v2019): Visualization of alignment

Recombination Detection Program (v4, RDP4) and Simplot (v3.5.1): recombination analysis and similarity plot visualization

SWISS-MODEL server (https://swissmodel.expasy.org/): spike protein RBD structure prediction.

For manuscripts utilizing custom algorithms or software that are central to the research but not yet described in published literature, software must be made available to editors/reviewers. We strongly encourage code deposition in a community repository (e.g. GitHub). See the Nature Research guidelines for submitting code \& software for further information. 
Policy information about availability of data

All manuscripts must include a data availability statement. This statement should provide the following information, where applicable:

- Accession codes, unique identifiers, or web links for publicly available datasets

- A list of figures that have associated raw data

- A description of any restrictions on data availability

The whole genome sequence obtained in this study was submitted to GenBank with the accession number MN908947.

Fig. 1-3, Extended Data Fig. 3, Extended Data Fig. 5-9 have associated raw data.

\section{Field-specific reporting}

Please select the one below that is the best fit for your research. If you are not sure, read the appropriate sections before making your selection. $\bigotimes$ Life sciences $\quad \square$ Behavioural \& social sciences $\quad \square$ Ecological, evolutionary \& environmental sciences

For a reference copy of the document with all sections, see nature.com/documents/nr-reporting-summary-flat.pdf

\section{Life sciences study design}

All studies must disclose on these points even when the disclosure is negative.

Sample size

The goal of this study was to find out the possible aetiologic agents associated with the severe respiratory disease occurred recently in the city of Wuhan, Hubei province, China. We studied one patient, and collected bronchoalveolar lavage fluid (BALF) from him who exhibited severe respiratory syndrome including fever, dizzy and cough. Since it is a discovery study, the number of individuals is irrelevant to the conclusions drawn in the paper.

Data exclusions No data was excluded from the analyses.

Replication The de novo assembly of reads was performed using two programs.

The whole genome viral sequence obtained from read assembly was confirmed by PCR assays.

The results from phylogenetic and recombination analyses were confirmed by multiple runs.

Randomization Not applicable. The goal of this study was to find out the possible aetiologic agent associated with the severe respiratory disease occurred recently in the city of Wuhan, Hubei province, China. Since we could obtain the BALF sample from only one patient who exhibited severe respiratory syndrome including fever, dizzy and cough, hence, randomization was not applicable to this study.

Blinding

Not applicable. Only one RNA library was generated in this study and thus no group allocation was performed.

\section{Reporting for specific materials, systems and methods}

We require information from authors about some types of materials, experimental systems and methods used in many studies. Here, indicate whether each material, system or method listed is relevant to your study. If you are not sure if a list item applies to your research, read the appropriate section before selecting a response.

Materials \& experimental systems

\begin{tabular}{l|l}
\hline$n / a$ & Involved in the study \\
$\square$ & $\square$ Antibodies \\
$\searrow$ & $\square$ Eukaryotic cell lines \\
$\square$ & $\square$ Palaeontology \\
$\square$ & $\square$ Animals and other organisms \\
$\square$ & $\square$ Human research participants \\
$\square$ & $\square$ Clinical data
\end{tabular}

\begin{tabular}{l|l}
\multicolumn{2}{l}{ Methods } \\
\hline n/a & Involved in the study \\
$\searrow$ & $\square$ ChIP-seq \\
$\searrow$ & $\square$ Flow cytometry \\
$\searrow$ & $\square$ MRI-based neuroimaging
\end{tabular}

\section{Human research participants}

Policy information about studies involving human research participants

Population characteristics Recently, a severe respiratory disease emerged in the city of Wuhan, Hubei province of China. The aim of this study is to find out the etiologic agent. Although clinic records from seven patients were available in this study, BAFL sample was only obtained from one patient. Herein, only one patient was described in the text based on the comments by Referees.

Recruitment

The patient who exhibited clinic signs of respiratory disease including fever and cough was recruited. 
